\title{
A Study of Dose Painting Radiotherapy Guided by Three-Dimensional Arterial Spin labeling Perfusion Magnetic Resonance Imaging for Non- Enhancing Low-Grade Gliomas
}

\section{Zihong Zhu}

The Affiliated Hospital of Southwest Medical University

\section{Guanzhong Gong}

Shandong Cancer Hospital: Shandong Cancer Hospital and Institute

\section{Lizhen Wang}

Shandong Cancer Hospital: Shandong Cancer Hospital and Institute

\section{Ya Su}

Shandong Cancer Hospital: Shandong Cancer Hospital and Institute

\section{Xiaohang Qin}

Shandong Cancer Hospital: Shandong Cancer Hospital and Institute

\section{Mingming Chen}

Shandong Cancer Hospital: Shandong Cancer Hospital and Institute

Jie Lu

Shandong Cancer Hospital: Shandong Cancer Hospital and Institute

\section{Yong Yin ( $\nabla$ yinyongsd@126.com )}

The Affiliated Hospital of Southwest Medical University

\section{Research}

Keywords: low-grade gliomas, non-enhancing, three-dimensional arterial spin labeling, dose painting, radiotherapy

Posted Date: November 3rd, 2021

DOI: https://doi.org/10.21203/rs.3.rs-1022697/v1

License: (1) This work is licensed under a Creative Commons Attribution 4.0 International License. Read Full License 


\section{Abstract}

Background: The purpose of this study was to investigate the feasibility and dosimetric characteristics of dose painting for non-enhancing low-grade gliomas (NE-LGGs) guided by threedimensional arterial spin labeling (3D-ASL) perfusion magnetic resonance imaging.

Methods: Eighteen patients with NE-LGGs were enrolled. 3D-ASL, T2 fluid attenuated inversion recovery (T2 FLAIR) and contrast-enhanced T1-weight magnetic resonance images were obtained. The gross tumor volume (GTV) was delineated on the T2 FLAIR. The hyper-perfusion region of the GTV (GTV-ASL) was determined by 3D-ASL, and the GTV-SUB was obtained by subtracting the GTV-ASL from the GTV. The planning target volume (PTV), PTV-ASL and PTV-SUB were developed by expanding the external margins of the GTV, GTV-ASL and GTV-SUB, respectively. Three plans were established for each patient: in plan 1, the traditional homogeneous prescription dose to the PTV was 45-60 Gy; in plan 2 and plan 3, the dose to the PTV-ASL increased by $10-20 \%$ based on plan 1, without the maximum dose constraint to the PTV-ASL in plan 3. The dosimetric differences among the three plans were compared.

Results: Compared with plan 1, the dose to $2 \%\left(D_{2} \%\right), 98 \%\left(D_{98 \%}\right)$ and $50 \%\left(D_{\text {mean }}\right)$ of PTV-ASL volumes increased by $14.67 \%, 16.17 \%$ and $14.31 \%$ in plan 2 and $19.84 \%, 15.52 \%$ and $14.27 \%$ in plan 3 , respectively $(P<0.05)$; the $D_{2 \%}$ of the PTV, PTV-SUB increased by $11.89 \%$ and $8.34 \%$ in plan $2,15.89 \%$ and $8.49 \%$ in plan 3 , respectively $(P<0.05)$. The $P T V$ coverages were comparable among the three plans $(P>0.05)$. In Plan 2 and plan 3, the conformity indexs decreased by $18.60 \%$ and $12.79 \%$; while the homogeneity indexs increased by 1.43 and 2 times $(P<0.05)$. Compared with plan 1 , the D $0.1 \mathrm{cc}$ of brain stem and Dmax of optic chiasma slightly increased in plan 2 and plan 3 , but the absolute doses met the dose constraint. The other organs at risk were similar among the three plans $(P>0.05)$.

Conclusions: The sub-volume with hyper-perfusion for NE-LGG radiotherapy can be segmented based on the perfusion difference guided by 3D-ASL. And the radiation dose to the hyper-perfusion area increased safely by $10-20 \%$ through dose painting.

\section{Background}

Low-grade gliomas (LGGs) are the most common brain tumors in children, accounting for approximately $15 \%$ of all gliomas [1, 2]. The most common treatment method for LGGs is maximum surgical resection, followed by postoperative adjuvant chemotherapy and other comprehensive treatments. However, radiotherapy is usually the first option for patients who are unable to undergo surgery. Both postoperative adjuvant radiotherapy and radical radiotherapy play an important role in the treatment of LGGs.

Nevertheless, there is much disagreement about how to determine tumor targets in radiotherapy for nonenhancing low-grade gliomas (NE-LGGs) with computed tomography (CT) or magnetic resonance

imaging (MRI). The vascular wall components of NE-LGGs are similar to those of healthy brain tissue. NELGGs have an intact blood-brain barrier, and the macromolecular contrast agent cannot pass the blood- 
brain barrier. The lesion appears on CT images as hypodense and on T1-and T2-weighted (T1W and T2W) MRI as hypo- and hyperintense areas, respectively, with visible edemas [3, 4]. In traditional methods, in order to ensure the target coverage of NE-LGGs radiotherapy, the abnormally hyperintense area (edema area) on T2 fluid attenuated inversion recovery (T2 FLAIR) is usually used as the standard for the delineation of tumor target region, which often has too large range and increases the risk of radiation damage to surrounding healthy tissues. Tatekawa et al. [5] found that there was no significant correlation between the high signal volume on FLAIR and overall survival (OS). Therefore, accurate delineation of tumor targets for NE-LGGs is critical for improving the curative effect and reducing injury.

As a non-invasive blood perfusion imaging technology, three-dimensional arterial spin labeling (3D-ASL) generates an image by magnetically labeling water molecules as an endogenous tracer as they travel through blood. Imaging does not require the destruction of the blood-brain barrier and can reflect microscopic changes in tissue blood perfusion and micro-vessel density [6, 7]. 3D-ASL perfusion imaging can effectively distinguish intracranial tumors from non-neoplastic lesions, and the tumor cerebral blood flow (CBF) value is significantly negatively correlated with survival [8]. Teng et al. showed that glioma puncture guided by 3D-ASL had a higher success rate than conventional MRI. In this study, 6 cases of low-enhancing or non-enhancing gliomas were successfully punctured, indicating that 3D-ASL has great potential in guiding the determination of tumor targets for NE-LGGs [9].

3D-ASL is widely used in the diagnosis, grading and efficacy evaluation of gliomas $[8,10,11]$, while its application in guiding NE-LGG radiotherapy has rarely been reported. Therefore, we studied the segmentation of tumor sub-volumes for NE-LGGs and dose painting of individualized radiotherapy based on 3D-ASL.

\section{Materials And Methods}

\section{Patient characteristics}

A total of 18 patients that had been pathologically diagnosed with LGGs at Shandong Cancer Hospital from December 2018 to May 2021 were retrospectively analysed. These patients showed no enhancement on contrast-enhanced T1-weighted MR images. The ages of the patients ranged from 5 to 74 years, with an average age of $42 \pm 21$ years, and there were $12(67 \%)$ males and $6(33 \%)$ females. Patient characteristics are presented in Table 1 (included at the end of the document text file).

Table 1 Clinical characteristics of the patients 


\begin{tabular}{|c|c|}
\hline Number of patients & 18 \\
\hline \multicolumn{2}{|l|}{ Gender } \\
\hline Male & $12(67 \%)$ \\
\hline Female & $6(33 \%)$ \\
\hline Age, mean \pm standard deviation & $42 \pm 21$ \\
\hline Male, mean \pm standard deviation & $43 \pm 21$ \\
\hline Female, mean \pm standard deviation & $40 \pm 21$ \\
\hline \multicolumn{2}{|l|}{ Age phases } \\
\hline Adults ( $\geq 18$ years) & $15(83 \%)$ \\
\hline Children (<18 years) & $3(17 \%)$ \\
\hline \multicolumn{2}{|l|}{ Tumor location } \\
\hline Unilateral & $7(39 \%)$ \\
\hline Midline & $8(44 \%)$ \\
\hline Bilateral (diffuse distribution) & $3(17 \%)$ \\
\hline \multicolumn{2}{|l|}{ Type of histopathology } \\
\hline Astrocytoma & $18(100 \%)$ \\
\hline \multicolumn{2}{|l|}{ WHO classification* } \\
\hline Grade II & $18(100 \%)$ \\
\hline \multicolumn{2}{|l|}{ IDH mutation status } \\
\hline Mutant & $1(6 \%)$ \\
\hline Wild type & $13(72 \%)$ \\
\hline Unclear (NOS) & $4(22 \%)$ \\
\hline \multicolumn{2}{|l|}{$1 p / 19 q$ deletion status } \\
\hline Codeletion & $0(0 \%)$ \\
\hline No deficiencies & $4(22 \%)$ \\
\hline Unclear & $14(78 \%)$ \\
\hline \multicolumn{2}{|c|}{ Concurrent temozolomide chemoradiotherapy } \\
\hline Yes & $14(78 \%)$ \\
\hline No & $4(22 \%)$ \\
\hline
\end{tabular}


* Grading of all enrolled patients according to the 2016 WHO classification of tumors of the central nervous system.

$\mathrm{IDH}$, isocitrate dehydrogenase.

Our retrospective study followed medical ethics guidelines, and the Institutional Review Board of Shandong Cancer Hospital approved this study. All patients signed informed consent forms. We protected patient privacy and excluded patient identification information from our analysis.

\section{Simulation}

CT simulation: For simulations, a CT scanner (Brilliance Big Bore, Philips, Netherlands) with a slice thickness of $3 \mathrm{~mm}$ and an interslice gap width of $3 \mathrm{~mm}$ was used, scanning from the foramen magnum to the end of the skull in a caudocranial direction.

MRI simulation: All MR images (T2 FLAIR, 3D-ASL and contrast-enhanced T1-weighted (CE-T1W)) were acquired with the same 3.0T MR scanner (Discovery 750W, GE Healthcare, USA) using a standard 6channel cranial coil. For 3D-ASL, the special acquisition conditions were as follows: number of excitations (NEX): 3; labeling duration: 1.5 s; post-Labeling delay time (PLD): 2025 ms. For CE-

T1W images, gadopentetate dimeglumine was power-injected at $2 \mathrm{~mL} / \mathrm{s}$ at doses standardized by patient body weight $(0.2 \mathrm{~mL} / \mathrm{kg}$ body weight), and the scan was started $3-5$ min after the injection. The other scan parameters for each sequence are described in Table 2.

Table 2 MRI simulated parameters for each sequence

\begin{tabular}{|llll|}
\hline Parameters & T2/FLAIR & 3D-ASL & CE-T1WI \\
\hline TR $(\mathrm{ms})$ & 11000 & 5160 & 8.5 \\
\hline TE $(\mathrm{ms})$ & 120 & 11.5 & 3.2 \\
\hline Layer thickness $(\mathrm{mm})$ & 3 & 3 & 3 \\
\hline FOV $(\mathrm{cm})$ & 26 & 25.6 & 25.6 \\
\hline Matrix & $320 \times 320$ & $512 \times 512$ & $256 \times 256$ \\
\hline
\end{tabular}

T2 FLAIR, T2 fluid attenuated inversion recovery; CE-T1WI, contrast-enhanced T1-weighted imaging; 3DASL, three dimensional arterial spin labeling; TR, repetition time; TE, echo time; FOV, field of view.

\section{Target volume definition}

All MRI images were imported into MIM Maestro software (6.8.8, USA). Two senior radiologists delineated the tumor target volume and organs at risks (OARs), and another senior radiologist confirmed the target 
region and modified it as needed. Axial T2 FLAIR sequences were used to contour the hyperintense signal abnormality as the gross tumor volume (GTV). The GTV-ASL was defined as a high perfusion region under the guidance of 3D-ASL sequence that was free of cysts, necrosis, calcifications, haemorrhage, and large vessels. The GTV-SUB was obtained by subtracting the GTV-ASL from the GTV.

The planning target volume (PTV) and PTV-ASL were generated by adding a 0.5 and $0.3 \mathrm{~cm}$ margin to GTV and GTV-ASL, respectively. The PTV-SUB was obtained by subtracting the PTV-ASL from the PTV. OARs included the bilateral eyeballs, bilateral lenses, bilateral optic nerves, optic chiasma and brain stem. A schematic diagram is shown in Fig. 1.

\section{Treatment planning}

All treatment plans were designed in the Eclipse (Version 15.6, Varian, USA) treatment planning system using intensity-modulated radiotherapy (IMRT). Three plans were designed for each patient, including plan 1 for conventional treatment and plan 2 and plan 3 for dose painting. In plan 1, patients were prescribed a traditional homogeneous dose of 45-60 Gy to the PTV, with a maximum dose constraint of $110 \%$ of the prescription dose, normalized to the PTV average dose. In plan 2, the dose of PTV-ASL increased by $10-20 \%$ based on plan 1 , with a maximum dose constraint of $110 \%$ of the dose to PTV-ASL. The plan 3 was obtained from plan 2 without the maximum dose constraint. The dose normalizations were set to the PTV-ASL average dose in plans 2 and plan 3.

The field angles were kept constant for all three treatment plans. Dose calculation was performed in anisotropic analytical algorithm optimization mode (AAA, version 15.512) using 6 MV X-rays. The calculated grid size was $2.5 \mathrm{~mm} \times 2.5 \mathrm{~mm}$, and the prescription covered $95 \%$ of the target volume.

The doses of OARs were restricted as following: brain stem D0.1cc $\leq 54 \mathrm{~Gy}$, eyeballs Dmax $<45 \mathrm{~Gy}$, lens Dmax $<10$ Gy, and optic nerves and optic chiasm Dmax $<55$ Gy (see Fig. 2 (A) and Fig. 2 (B)).

\section{Plan evaluation}

To compare the three treatment plans, the dose distribution and dose-volume histograms (DVHs) of the PTV, PTV-SUB, PTV-ASL and OARs were calculated. The $\mathrm{D}_{2 \%}$ and $\mathrm{D}_{98 \%}$ (where Dx indicates the dose to $\mathrm{x} \%$ of the target volume) as well as $\mathrm{D}_{\text {mean }}$ were compared for the PTV, PTV-SUB and PTV-ASL. Furthermore, the target coverage, conformity index $(\mathrm{Cl})$ and homogeneity index $(\mathrm{HI})$ were calculated. A Cl close to 1 indicates that the shape of the region receiving the reference dose closely matches the shape of the target region, and an $\mathrm{HI}$ close to 0 indicates good uniformity. The formulas for calculating $\mathrm{Cl}$ and $\mathrm{HI}$ are as follows: $\mathrm{Cl}=(\mathrm{Vt}, \mathrm{ref} / \mathrm{Vt}) \times(\mathrm{Vt}$,ref/Vref $)$, where $\mathrm{Vt}$ represents the volume of the target region, $\mathrm{Vt}$,ref represents the volume of the target region covered by the reference dose, and Vref represents the volume of all regions covered by the reference dose; and $H I=\left(D_{2 \%}-D_{98 \%}\right) / D_{50 \%}$. The flowchart is available in Fig. 3.

\section{Statistical analysis}


Statistical analyses were performed using IBM SPSS version 25.0. Comparisons between two groups were done with paired $t$-tests or Wilcoxon tests, depending on the distribution defined by the Shapiro-Wilk (S-W) normality test. Comparisons of multiple groups were performed by 2-way analysis of variance (ANOVA), followed by least significant difference (LSD) and Friedman two-way ANOVA, followed by Friedman multiple comparison tests, depending on Levene's test of variance homogeneity. All data are expressed as $\overline{\mathrm{x}} \pm \mathrm{s}$ (mean \pm standard deviation), and $P<0.05$ indicates a significant difference.

\section{Results}

\section{Comparison of target volumes and imaging findings}

The average volume was $42.49 \mathrm{~cm}^{3}$ for GTV-ASL in comparison with $152.92 \mathrm{~cm}^{3}$ for GTV, resulting in a reduction of $72.21 \%(Z=-3.724, P=0.000)$. And the average volume was $90.06 \mathrm{~cm}^{3}$ for PTV-ASL in comparison with $383.95 \mathrm{~cm}^{3}$ for PTV, resulting in a reduction of $76.54 \% \%(Z=-3.724, P=0.000)$. Evident high perfusion was detected within edema areas in all patients. Figure. 4 shows a representative patient with different $M R$ sequences.

\section{Comparison of $D_{2 \%}, D_{98 \%}$ and $D_{\text {mean }}$ among the three treatment plans}

(1) The doses of the PTV, PTV-SUB and PTV-ASL increased in plan 2 and plan 3 than plan 1. The $\mathrm{D}_{2 \%}$ increased by $11.89 \%, 8.34 \%$, and $14.67 \%$ in plan 2 and $15.89 \%, 8.49 \%$, and $19.84 \%$ in plan 3 , respectively, with statistically significant differences $(P<0.05)$; the $\mathrm{D}_{98 \%}$ increased by $1.12 \%, 0.93 \%$, and $16.17 \%$ in plan 2 and $0.7 \%, 0.49 \%, 15.52 \%$ in plan 3 , respectively, but only increased significantly for the PTV-ASL in plans 2 and $3(P<0.05)$; and $D_{\text {mean }}$ increased by $5.15 \%, 3.60 \%$, and $14.31 \%$ in plan 2 and $4.62 \%, 2.71 \%$, and $14.27 \%$ in plan 3 , respectively, with statistically significant differences $(P<0.05)$ except for the PTV in plan 3.

(2) Compared with plan 2, the $\mathrm{D}_{2 \%}$ of the PTV, PTV-SUB and PTV-ASL increased by $3.58 \%, 0.51 \%$ and $4.52 \%$ in plan 3 , respectively, with statistically significant differences for the PTV and PTV-ASL $(P<0.05)$; $D_{98 \%}$ and $D_{\text {mean }}$ decreased by $0.41 \%, 0.43 \%$, and $0.56 \%$ and $0.50 \%, 0.86 \%$, and $0.03 \%$, respectively, with no statistically significant differences $(P>0.05)$.

(3) In the PTV-ASL, the $\mathrm{D}_{2 \%}, \mathrm{D}_{98 \%}$ and $\mathrm{D}_{\text {mean }}$ increased by at least $10 \%$ in plan 2 and plan 3 than plan 1 , with the most significant increase of $\mathrm{D}_{2 \%}$ in plan 3 at $19.84 \%$, as shown in Table 3 (provided as an additional file) and Fig. 5.

\section{Plan evaluation}

The PTV target coverages for plan 1, plan 2 and plan 3 were comparable $(P>0.05)$. Compared with plan 1 , the Cls decreased by $18.60 \%$ in plan 2 and $12.79 \%$ in plan 3, respectively, with statistically significant differences $(P<0.05)$, but the Cls were comparable between plan 2 and plan $3(P>0.05)$. Compared with 
plan 1, the Hls increased by 1.43 in plan 2 and 2 times in plan 3, respectively; and in increased by $23.53 \%$ in plan 3 than in plan $2(P<0.05)$.

\section{Dosimetric Comparison of OARs}

The Dmax of the bilateral eyeballs, bilateral lens, and bilateral optic nerves were comparable among plan 1 , plan 2, and plan $3(P>0.05)$. Compared to plan 1 , the D0.1 cc of brain stem and the Dmax of optic chiasma were slightly increased by $3.98 \%$ and $4.52 \%$ in plan 2 and $4.33 \%$ and $6.27 \%$ in plan 3 , respectively, with statistically significant differences $(P<0.05)$, but all absolute doses were safe. And they were comparable between plan 2 and plan 3 ( $P>0.05$ ), as shown in Table 4 (provided as an additional file).

\section{Discussion}

The main innovation of this study is the demonstration that MR 3D-ASL images can be used to quantify and analyse tumor perfusion differences in NE-LGGs, and that sub-volume segmentation of the GTV can be performed based on this perfusion difference, allowing for safe dose escalation in hyper-perfused regions by the boost technique.

LGGs grow slowly and have a long disease duration, and most LGGs inevitably develop into high-grade malignant gliomas, with an incidence of malignant transformation of up to $17 \%$ and a poor prognosis, with a median overall survival time of only 2.4 years after malignant transformation [12-14].

Radiotherapy is an effective treatment for LGGs, allowing for the control of primary tumor lesions and improving patient survival. However, failure of local control remains the predominant cause of LGG treatment failure. Most LGG recurrences after radiotherapy predominantly occur in the radiation field in conventional radiotherapy, which is significantly associated with insufficient doses to the tumor target region $[15,16]$. Prospective clinical trials, such as EORTC 22844 and NCCTG 86-72-51, showed that highdose radiotherapy did not improve the survival of patients with LGGs, but rather increased the risk of radiation necrosis and reduced the quality of life [17-19]. However, Liu et al. [20] demonstrated the survival benefits of high-dose radiotherapy in patients with IDH wild-type (IDHwt) LGGs. In this study, 52 patients with IDHwt LGGs treated with radiotherapy were divided into two groups: a high-dose group with doses $>54$ Gy and a low-dose group with doses $\leq 54 \mathrm{~Gy}$. The results showed that high-dose radiotherapy was associated with increased survival rates. Liu et al. [21] showed that high-dose radiotherapy (>54 Gy) was an independent prognostic factor of LGGs and was associated with an increase in the survival rate of patients with 06-methylguanine-DNA methyltransferase promoter nonmethylation (MGMT-non-pM). Therefore, increasing the radiation dose can improve the survival rates of patients with special molecular subtypes of LGGs. And in this study, more than $70 \%$ the participants were patients with IDHwt LGGs, so we conducted the study of escalation.

The EORTC 22844 and NCCTG 86-72-51 trials were mainly based on conventional anatomical MR images for homogeneous dose boosting, with a simultaneous dose increase for healthy tissues and an inevitable increase in radiation damage. In contrast, dose painting radiotherapy guided by functional imaging, such 
as functional MR and PET, is expected to achieve a safe dose boost. Kim et al. [22] investigated the region of hyperperfused/hypercellular $\left(\mathrm{TV}_{\mathrm{HCV}} / \mathrm{TV}_{\mathrm{CBV}}\right)>1 \mathrm{~cm}^{3}$ in high b-value diffusion-weighted MRI and dynamic enhanced perfusion MRI (DCE) for glioblastoma with dose boosting (75 Gy/30 f) and found that it resulted in good survival rates (12-month OS rate of $92 \%(P=0.03)$ and median OS of 20 months) with fair neurocognitive function and quality of life.

NE-LGGs, as a group of tumors with significant heterogeneity, require a more individualized radiotherapy dose to be applied to the tumor target region. NE-LGGs have an intact blood-brain barrier and do not show enhancement on CT or MRI. There is considerable blindness when the GTV is delineated with the edema area as the main criterion. Jakola et al. [23] showed that the blood-brain barriers of NE-LGGs were disrupted after malignant transformation, and the region that appeared to be enhanced was much smaller than the edema area. Although coverage of the radiotherapy dose to the tumor can be guaranteed if the edema area is directly defined as the GTV range of the NE-LGG, it is difficult to increase the radiotherapy dose due to the large irradiation range. Although it is difficult to define tumor margins in imaging without enhanced LGGs, there are still significant biological differences between the blood flow and metabolism of the tumor and the brain tissue in the edema area [24]. Quantifying this difference with functional imaging can clarify the distribution of tumor cell-enriched areas, which will provide a reliable reference for the heterogeneous distribution of radiotherapy doses.

3D-ASL is used for the brain tumors diagnosis and radiotherapy efficacy/toxicity predictions by labeling water molecules for perfusion imaging of tissues, effectively avoiding the risks of exogenous contrast agents [6]. Related studies have shown that the CBF determined by 3D-ASL is significantly positively correlated with micro-vessel density and vascular endothelial growth factor expression ( $r=0.604)$ but significantly negatively correlated with survival $(r=-0.714)$ and can be an independent risk factor for OS $(H R=1.028)[25,26]$. This suggests that dose boosting in regions of high CBF in LGG radiotherapy is necessary.

We found that NE-LGGs showed no abnormalities on MR-enhanced T1 but showed local high perfusion on 3D-ASL. And all the local hyper-perfusion regions were within edema areas through the fusion images of T2 FLAIR and 3D-ASL. We also found that the volume of the hyper-perfusion region (GTV-ASL) was significantly smaller than the volume of the edema area (GTV) and by volume analysis of each target region. Therefore, boosting the local hyper-perfusion sub-volume region of 3D-ASL, the target volume of dose boosting was reduced, so as to the risk of radiation damage to surrounding healthy brain tissue.

In this study, plan 1 is the standard plan and plan 2 and plan 3 are dose painting plans. Compared with plan 1 , the $D_{2 \%}, D_{98 \%}$ and $D_{\text {mean }}$ of the PTV-ASL increased by more than $14 \%$ in plan 2 and plan 3; however, these values increased by less than $9 \%$ in the PTV-SUB, with $D_{98 \%}$ increased by less than $1 \%$. This suggests that under the guidance of 3D-ASL, the dose painting plan can achieve a significant dose boost in hyper-perfused sub-volume region for NE-LGGs while having less impact on the peripheral dose and avoiding dose boost blindness. This is similar to the study of Thureau et al., which was based on ${ }^{18}$ FDG PET-CT image-guided dose boost for locally advanced non-small-cell lung cancer (NSCLC) [27]. On 
the basis of not increasing the dose to OARs, the dose to high recurrence risk areas increased by $12.12 \%$, whereas the doses to other areas of the GTV only increased by $2.88 \%$. We also found that the $D_{2 \%}$ of the PTV, PTV-SUB and PTV-ASL gradually increased in plan 1, plan 2 and plan 3, whereas the $D_{98 \%}$ and $D_{\text {mean }}$ of each target region were comparable between plan 2 and plan 3 . Therefore, the elimination of maximum dose constraints is favourable to boost the maximum dose but has no significant effect on the minimum or average dose.

The target coverages of the PTV were comparable among plan 1, plan 2 and plan 3, with the prescribed isodose line wrapping around approximately $95 \%$ of the PTV, indicating that all treatment plans for plan 2 and plan 3 met the minimum dose requirements of the target region. Because only the local hyperperfused regions on 3D-ASL were escalated in plan 2 and plan 3, whereas the edema areas surrounding the hyper-perfused regions received standard doses, the HIs were larger in plan 2 and plan 3 than in plan 1. In this study, although the conformality and homogeneity of plan 2 and plan 3 decreased, the dose to the tumor target region was significantly increased. Therefore, reducing the homogeneity of the radiation dose is beneficial for dose escalation and protecting OARs.

During radiotherapy for patients with NE-LGGs, radiation damage to surrounding healthy tissues should not be ignored. The dose boost to the tumor target region must be balanced with protecting OARs. The results of this study showed that, compared with plan 1, the D0.1cc of brain stem and the Dmax optic chiasma increased by less than $5 \%$ in plan 2 and plan 3 , but they were within a safe range. The other OARs were comparable among the three treatment plans, which were consistent with the findings of Kosztyla et al. [28].

The main limitations of our study were as follows: (1) The small number of patients is due to the relatively low incidence rate of NE-LGGs, as well as the fact that the vast majority of patients underwent surgery and were excluded from analysis after surgery due to the disruption of the blood-brain barrier, which often shows enhancement on conventional enhanced MRI. (2) Because this is a preclinical technical study, it is necessary to increase the sample size and include the PFS and OS of patients to evaluate the value of this technique for further clinical applications.

\section{Conclusion}

It is feasible to segment sub-volume in NE-LGG radiotherapy based on the perfusion differences under the guidance of 3D-ASL. The radiation dose to highly perfused regions can be safely increased by $10-20 \%$ by dose painting without increasing the radiation dose to OARs, which is conducive for improving the survival rate of patients with NE-LGGs and provides a basis for future individualized and precise radiotherapy for NE-LGGs.

\section{Abbreviations}


NE-LGGs: non-enhancing low-grade gliomas; LGGs: low-grade gliomas; 3D-ASL: threedimensional arterialspin labeling; T2 FLAIR: T2 fluid attenuated inversion recovery; CE-T1W: contrastenhanced T1-weighted; T1W: T1-weighted; T2W: T2-weighted; CT: Computed Tomography; MRI: magnetic resonance imaging; GTV: the gross tumor volume; PTV: planning target volume; OARs: organs at risk; CBF: cerebral blood flow; WHO: world health organization; IDH: isocitrate dehydrogenase; IDHwt: isocitrate dehydrogenase wild-type; MGMT-non-pM: methylguanine-DNA methyltransferase promoter nonmethylation; NEX: number of excitation; PLD: post labeling delay time; TR: repetition time; TE: echo time; FOV: field of view; IMRT: intensity-modulated radiotherapy; AAA: anisotropic analytical algorithm optimization mode; Dx: the dose to $x \%$ target volume; D $0.1 \mathrm{cc}$ : the dose to $0.1 \mathrm{cc}$ volume; Dmax: the maximum dose; $\mathrm{D}_{\text {mean }}$ : the average dose; DVHs: dose-volume histograms; $\mathrm{Cl}$ : conformity index; $\mathrm{HI}$ : homogeneity index; ANOVA: analysis of variance; LSD: the least significant difference.

\section{Declarations}

\section{Acknowledgments}

Not applicable.

\section{Authors' Contributions}

Guanzhong Gong conceived and designed the study. Lizhen Wang and Ya Su collected data. Xiaohang Qin and Mingming Chen made a statistical analysis. Zihong zhu performed the experiments and wrote the paper. Yong Yin and Jie Lu reviewed and edited the manuscript. All authors read and approved the manuscript.

\section{Funding}

This work was supported by Cultivation Project of Shandong Cancer Hospital (Grant No. YYPY2020-016).

\section{Availability of data and materials}

The data set used and analysed during the current study were available from the corresponding author on reasonable request.

\section{Ethical approval and consent to participate}

This study was reviewed and approved by the Institutional Review Board of Shandong Cancer Hospital. All patients signed informed consent forms.

\section{Consent for publication}

Not applicable.

\section{Competing interests}


The authors declare that they have no competing interests.

\section{Author details}

${ }^{1}$ Department of Oncology, Affiliated Hospital of Southwest Medical University, No.25 Taiping Street, Jiangyang District, Luzhou City, 646000, Sichuan Province, China

2 Department of Radiation Oncology Physics and Technology, Shandong Cancer Hospital and Institute, Shandong First Medical University and Shandong Academy of Medical Sciences, No.440 Jiyan road, Huaiyin district, Jinan City, 250117, Shandong Province, China

\section{References}

1. Ostrom QT, Patil N, Cioffi G, et al. CBTRUS Statistical Report: Primary Brain and Other Central Nervous System Tumors Diagnosed in the United States in 2013-2017. Neuro Oncol, 2020;22(12 Suppl 2): iv1-iv96.

2. Nunna RS, Khalid S, Ryoo JS, et al. Radiotherapy in adult low-grade glioma: nationwide trends in treatment and outcomes. Clin Transl Oncol, 2021;23(3):628-637.

3. Upadhyay N, Waldman AD. Conventional MRI evaluation of gliomas. Br J Radiol. 2011;84 (Spec Iss 2): S107-S111.

4. Afra D, Osztie E. Histologically confirmed changes on CT of reoperated low-grade astrocytomas. Neuroradiology. 1997;39(11):804-810.

5. Tatekawa $\mathrm{H}$, Uetani $\mathrm{H}$, Hagiwara A, et al. Worse prognosis for IDH wild-type diffuse gliomas with larger residual biological tumor burden. Ann Nucl Med. 2021;35(9):1022-1029.

6. Furtner J, Bender B, Braun C, et al. Prognostic value of blood flow measurements using arterial spin labeling in gliomas. PLoS One. 2014;9(6): e99616.

7. Telischak NA, Detre JA, Zaharchuk G. Arterial spin labeling MRI: clinical applications in the brain. $J$ Magn Reson Imaging. 2015;41(5):1165-1180.

8. Soni N, Srindharan K, Kumar S, et al. Arterial spin labeling perfusion: Prospective MR imaging in differentiating neoplastic from non-neoplastic intra-axial brain lesions. Neuroradiol J. 2018;31(6):544-553.

9. Jin T, Ren Y, Zhang H, et al. Application of MRS- and ASL-guided navigation for biopsy of intracranial tumors. Acta Radiol. 2019;60(3):374-381.

10. Falk Delgado A, De Luca F, van Westen $D$, et al. Arterial spin labeling MR imaging for differentiation between high- and low-grade glioma-a meta-analysis. Neuro Oncol. 2018;20(11):1450-1461.

11. Xu Q, Liu Q, Ge H, et al. Tumor recurrence versus treatment effects in glioma: A comparative study of three-dimensional pseudo-continuous arterial spin labeling and dynamic susceptibility contrast imaging. Medicine (Baltimore). 2017;96(50): e9332. 
12. Lombardi G, Barresi V, Castellano A, et al. Clinical Management of Diffuse Low-Grade Gliomas. Cancers (Basel), 2020;12(10): 3008.

13. Obara T, Blonski M, Brzenczek C, et al. Adult Diffuse Low-Grade Gliomas: 35-Year Experience at the Nancy France Neuro-oncology Unit. Front Oncol, 2020;10: 574679.

14. Tom MC, Park DYJ, Yang K, et al. Malignant Transformation of Molecularly Classified Adult LowGrade Glioma. Int J Radiat Oncol Biol Phys, 2019;105(5): 1106-1112.

15. Jaspers JPM, Méndez Romero A, Wiggenraad R, et al. Pattern of failure in IDH mutated, low grade glioma after radiotherapy - Implications for margin reduction. Radiother Oncol. 2021; 156:43-48.

16. Kamran SC, Dworkin M, Niemierko A, et al. Patterns of Failure Among Patients with Low-grade Glioma Treated with Proton Radiation Therapy. Pract Radiat Oncol. 2019;9(4): e356-e361.

17. Karim AB, Maat B, Hatlevoll $R$, et al. A randomized trial on dose-response in radiation therapy of lowgrade cerebral glioma: European Organization for Research and Treatment of Cancer (EORTC) Study 22844. Int J Radiat Oncol Biol Phys. 1996;36(3):549-556.

18. Breen WG, Anderson SK, Carrero XW, et al. Final report from Intergroup NCCTG 86-72-51 (Alliance): a phase III randomized clinical trial of high-dose versus low-dose radiation for adult low-grade glioma. Neuro Oncol. 2020;22(6):830-837.

19. Prabhu RS, Ward MC, Heinzerling JH, et al. The Association Between Radiation Therapy Dose and Overall Survival in Patients with Intracranial Infiltrative Low-Grade Glioma Treated with Concurrent and/or Adjuvant Chemotherapy. Adv Radiat Oncol. 2020;6(1):100577.

20. Liu S, Liu Y, Li G, et al. High-dose radiation associated with improved survival in IDH-wildtype lowgrade glioma. Chin Neurosurg J. 2021;7(1):22.

21. Liu Y, Li Y, Wang P, et al. High-dose radiotherapy in newly diagnosed low-grade gliomas with nonmethylated O (6)-methylguanine-DNA methyltransferase. Radiat Oncol. 2021;16(1):157.

22. Kim MM, Sun Y, Aryal MP, et al. A Phase 2 Study of Dose-intensified Chemoradiation Using Biologically Based Target Volume Definition in Patients with Newly Diagnosed Glioblastoma. Int $J$ Radiat Oncol Biol Phys. 2021;110(3):792-803.

23. Jakola AS, Bouget D, Reinertsen I, et al. Spatial distribution of malignant transformation in patients with low-grade glioma. J Neurooncol. 2020;146(2):373-380.

24. Galldiks N, Niyazi M, Grosu AL, et al. Contribution of PET imaging to radiotherapy planning and monitoring in glioma patients - a report of the PET/RANO group. Neuro Oncol. 2021;23(6):881-893.

25. Ningning $D$, Haopeng $P$, Xuefei $D$, et al. Perfusion imaging of brain gliomas using arterial spin labeling: correlation with histopathological vascular density in MRI-guided biopsies [published correction appears in Neuroradiology. 2017 Feb;59(2):209]. Neuroradiology. 2017;59(1):51-59.

26. Pang $H$, Dang $X$, Ren $Y$, et al. 3D-ASL perfusion correlates with VEGF expression and overall survival in glioma patients: Comparison of quantitative perfusion and pathology on accurate spatial locationmatched basis. J Magn Reson Imaging. 2019;50(1):209-220. 
27. Thureau S, Dubray B, Modzelewski R, et al. FDG and FMISO PET-guided dose escalation with intensity-modulated radiotherapy in lung cancer. Radiat Oncol. 2018;13(1):208.

28. Kosztyla R, Raman S, Moiseenko V, et al. Dose-painted volumetric modulated arc therapy of highgrade glioma using 3,4-dihydroxy-6-[ $\left.{ }^{18} \mathrm{~F}\right]$ fluoro-L-phenylalanine positron emission tomography. $\mathrm{Br} \mathrm{J}$ Radiol. 2019;92(1099):20180901.

\section{Tables}

Due to technical limitations, table 3 and 4 is only available as a download in the Supplemental Files section.

\section{Figures}

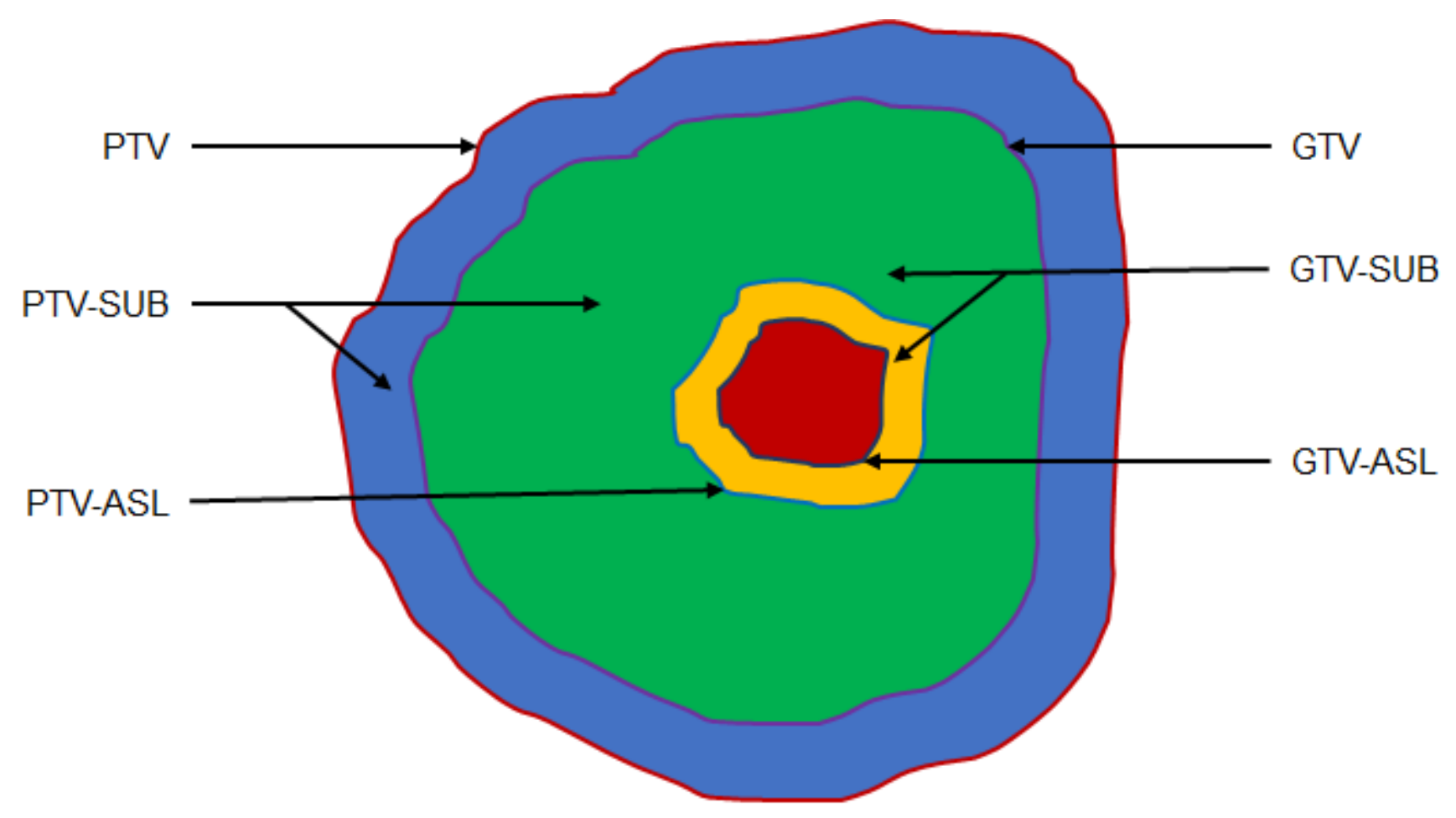

\section{Figure 1}

A schematic diagram of target volumes definition. GTV, gross tumor volume; GTV-ASL, the high perfusion region in the 3D-ASL sequence. The planning target volume (PTV) and PTV-ASL were generated by adding 0.5 and $0.3 \mathrm{~cm}$ margin to GTV and GTV-ASL, respectively. GTV-SUB and PTV-SUB were obtained by subtracting the GTV-ASL from the GTV and the PTV-ASL from the PTV. 

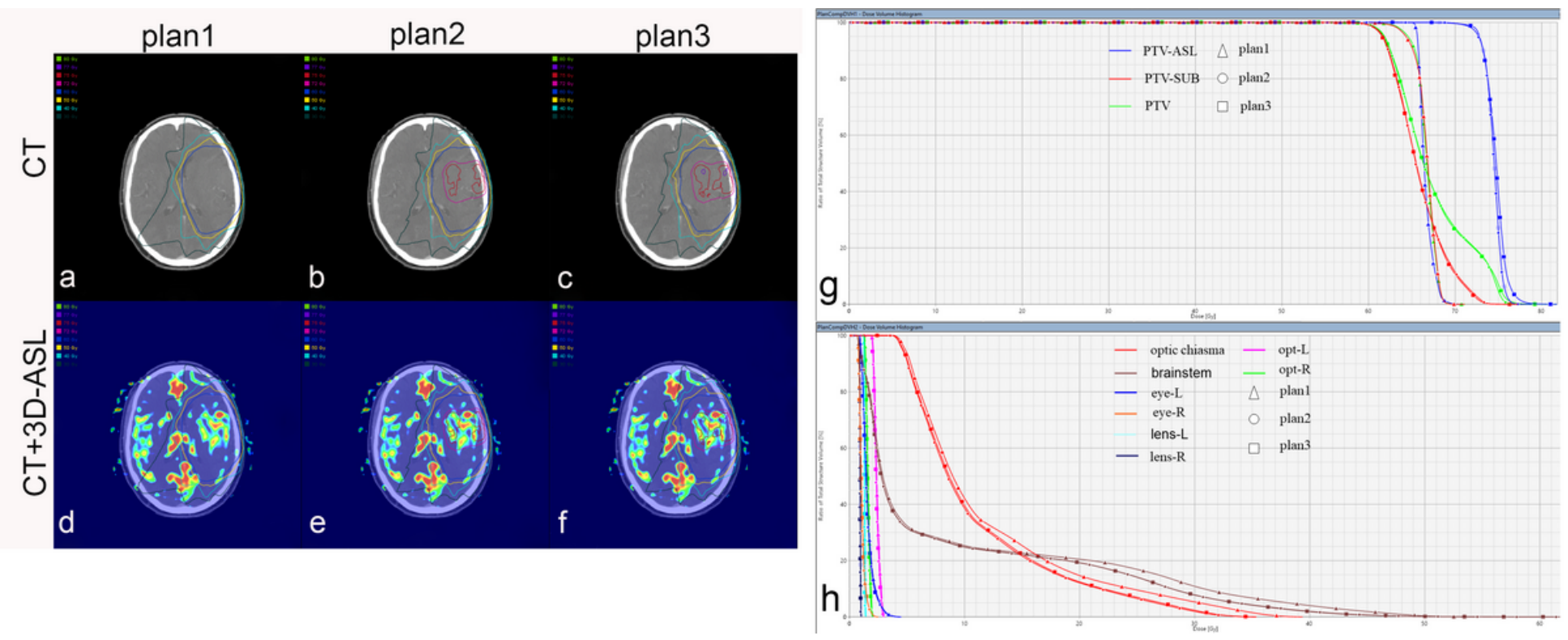

Figure 2

(A) The differences among conventional plan (plan 1) and dose painting plans (plan 2 and plan 3 ) are showed in this example. a and d (plan 1): prescribed a homogeneous dose of 60 Gy to the PTV; $b$ and $\mathrm{e}($ plan2), $\mathrm{c}$ and $\mathrm{f}$ (plan3): the dose of PTV-ASL increased by $20 \%$ (equivalent to $72 \mathrm{~Gy}$ ) based on plan 1 , without the maximum dose constraint in plan 3. a, b and c are based on computed tomography (CT); d, e and $f$ are based on the fusion images of CT and three-dimensional arterial spin labeling (3D-ASL). (B) The dose-volume histograms (DVHs) of three treatment plans. The DVHs of the target regions and organs at risks (OARs) are showed in $\mathrm{g}$ and $\mathrm{h}$, respectively. 


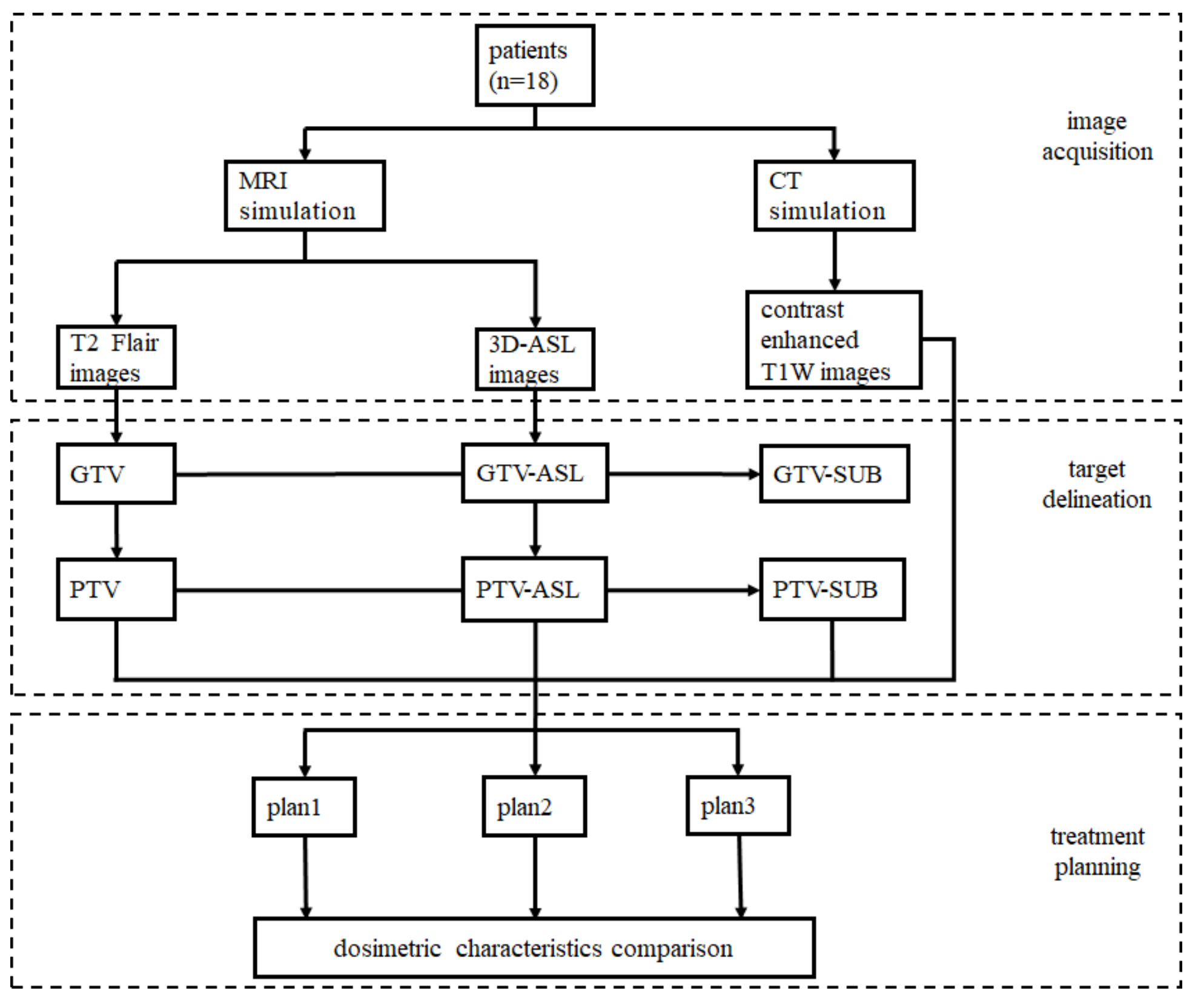

Figure 3

The working flow chart. 


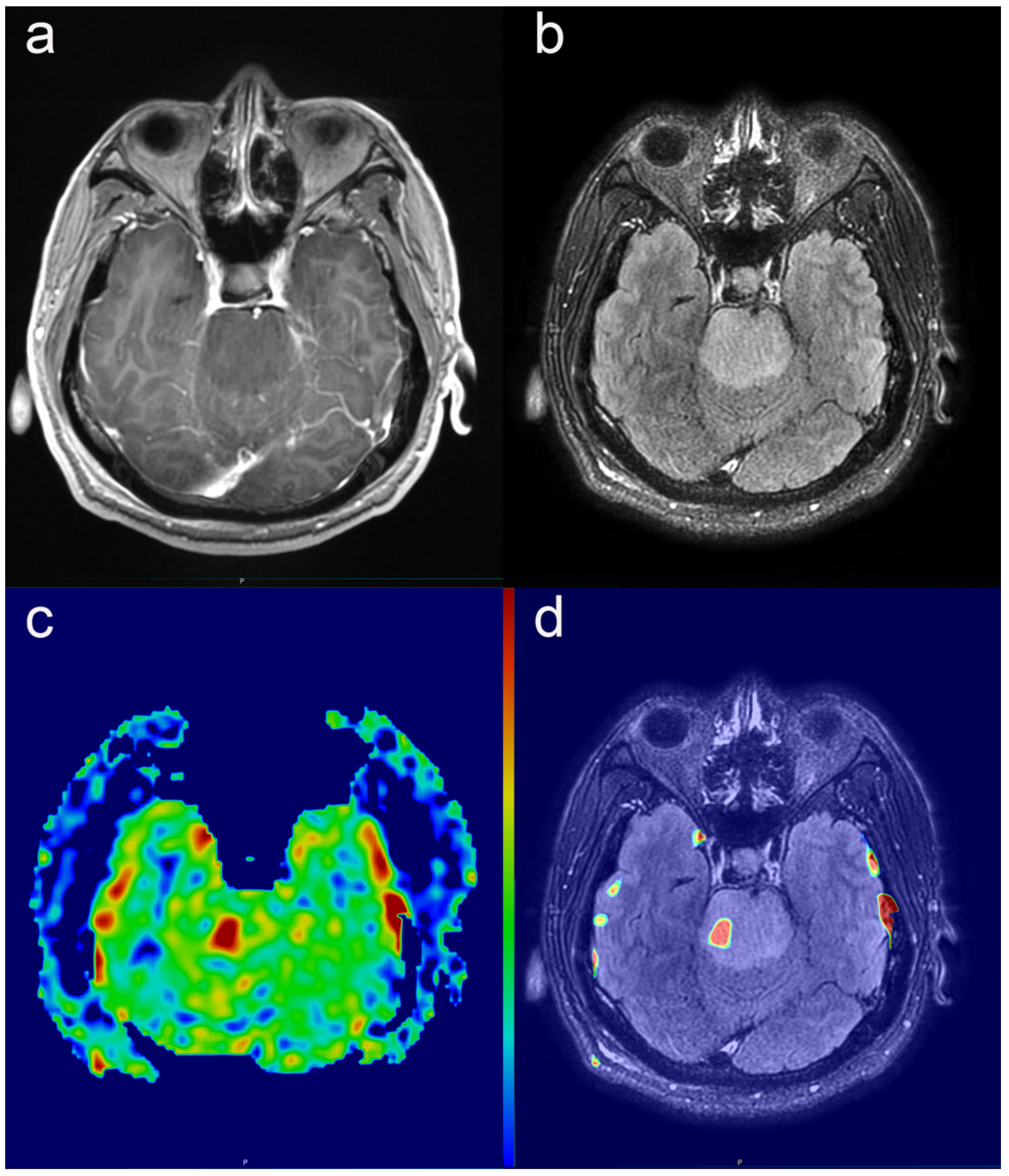

\section{Figure 4}

The exemplary imaging characteristics of tumor in different MR sequences. A 40 years old male was diagnosed with diffuse intrinsic pontine glioma (DIPG), WHO grade II. The image showed no abnormality on MR-enhanced T1-weighted (a), but appeared local hyper-perfusion on 3D-ASL (c), which was within the edema area $(b, d)$. b: T2 FLAIR; $d$ : The fusion image of T2 FLAIR and 3D-ASL. 


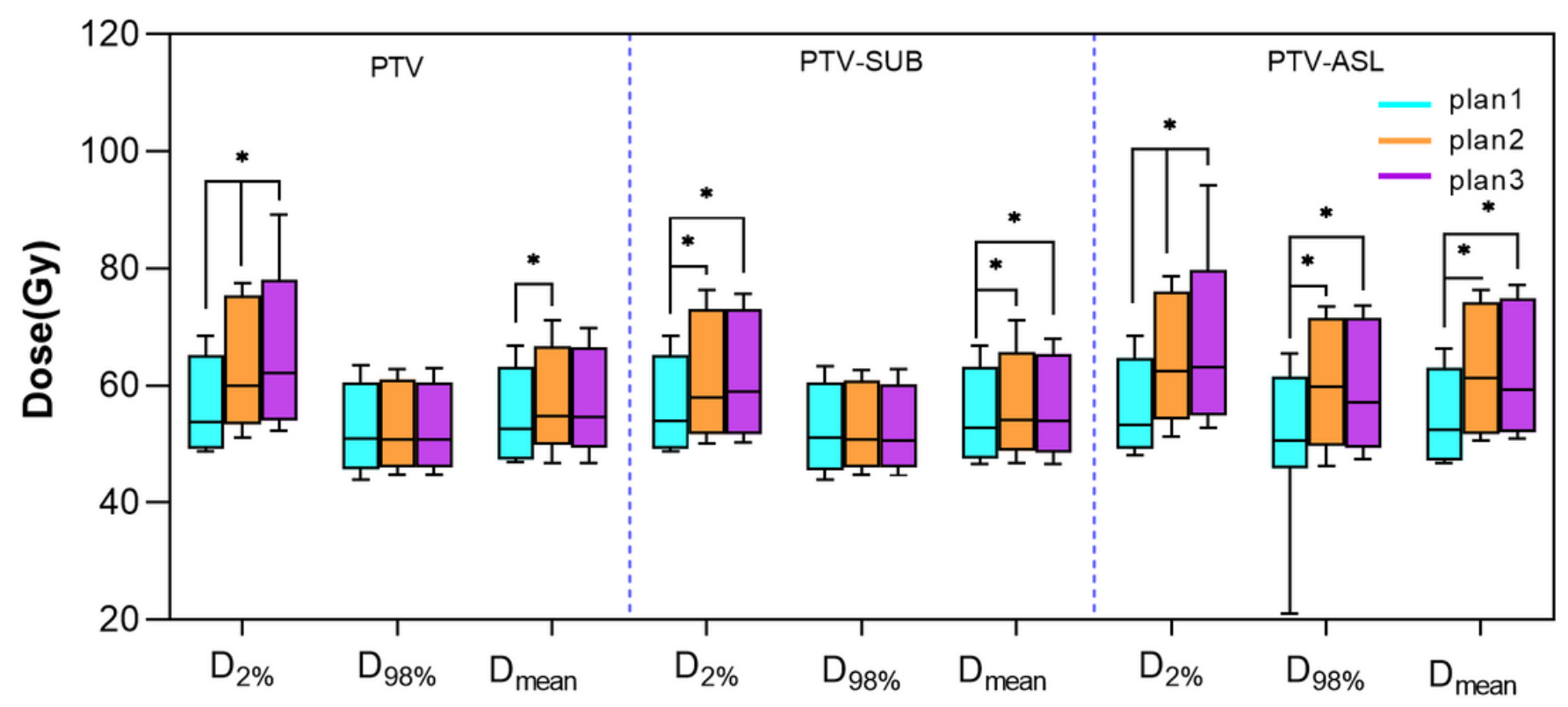

Figure 5

Dosimetric comparison of target regions among plan 1 (light blue), plan 2(orange) and plan 3 (purple). Compared with plan 1, the dose escalated more significantly in PTV-ASL than PTV-SUB in plan 2 and plan 3.

\section{Supplementary Files}

This is a list of supplementary files associated with this preprint. Click to download.

- Table3.xls.xlsx

- Table4.xls.xlsx 